\title{
Evaluation of a zinc chelate on clinical swine dysentery under field conditions
}

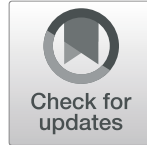

Frédéric Vangroenweghe ${ }^{1^{*}}$ (D) Liesbeth Allais ${ }^{2}$, Ellen Van Driessche ${ }^{2}$, Robbert van Berkel ${ }^{3}$, Gerwen Lammers $^{3}$ and Olivier Thas ${ }^{4,5,6}$

\begin{abstract}
Background: Brachyspira hyodysenteriae is the primary cause of swine dysentery, characterized by bloody to mucoid diarrhea due to mucohaemorhagic colitis in pigs and primarily affects pigs during the grow/finishing stage. Control and prevention of $B$. hyodysenteriae consists of administration of antimicrobial drugs, besides management and adapted feeding strategies. A worldwide re-emergence of the disease has recently been reported with an increasing number of isolates demonstrating decreased susceptibility to several crucially important antimicrobials in the control of swine dysentery. A novel non-antibiotic zinc chelate has been reported to demonstrate positive effects on fecal quality and consistency, general clinical signs, average daily weight gain and B. hyodysenteriae excretion during and after a 6-day oral treatment. The objective of the present study was to evaluate the zinc chelate (Intra Dysovinol $499 \mathrm{mg} / \mathrm{ml}$ (ID); Elanco) on naturally occurring swine dysentery due to B. hyodysenteriae under field conditions in the Netherlands.
\end{abstract}

Results: Oral administration of zinc chelate resulted in improvement of general clinical signs from 3 days onwards in the ID-treated group combined with a significantly better total fecal score at 14 days post-treatment. Overall, average daily weight gain was better in the ID-treated group over the entire study period (0-14 days) and during the 8 days following the end of ID-treatment. A significant reduction (4.48 vs. $0.63 \log _{10} \mathrm{cfu} / \mathrm{g}$ feces; ID-treated vs. control) in B. hyodysenteriae excretion was observed during the 6-day treatment period with a high percentage of animals (58.3 vs. $12.3 \%$; ID-treated vs. control) with no excretion of B. hyodysenteriae from their feces. No additional antimicrobial treatment was needed in the ID-treated group, whereas 35\% of the pigs in the control group were treated with an antibiotic at least once. No mortality occurred in both groups. No adverse events were reported during and following the ID-treatment.

Conclusions: Zinc chelate - administered as a Zn-Na2-EDTA complex - is a non-antibiotic treatment for swine dysentery that reduces $B$. hyodysenteriae shedding with $4.48 \log _{10} \mathrm{cfu} / \mathrm{g}$ feces within its 6 -day treatment while improving general clinical signs ( 90.0 vs. $73.6 \%$ animals with normal score) and total fecal score within $2-4$ days following administration in naturally infected pigs. The positive effects of ID treatment remain for at least 8 days after cessation of oral ID therapy. Pigs remaining in a highly contaminated environment may be re-infected following the end of ID treatment, however, this is not different to standard antimicrobial therapy. Therefore, control of swine dysentery should combine an efficacious treatment with additional management practices to reduce the environmental infection pressure in order to limit re-infection as much as possible. The ID treatment resulted in a higher growth rate and improved general health, whereas no mortality was observed and no additional therapeutic treatments were necessary in contrast to the control pigs.

Keywords: Zinc chelate, Brachyspira hyodysenteriae, Swine dysentery

\footnotetext{
* Correspondence: vangroenweghe.frederic@telenet.be

'Elanco, BU Food Animals, Plantijn en Moretuslei 1 - 3rd floor, 2018

Antwerpen, Belgium

Full list of author information is available at the end of the article
}

(c) The Author(s). 2020 Open Access This article is distributed under the terms of the Creative Commons Attribution 4.0 International License (http://creativecommons.org/licenses/by/4.0/), which permits unrestricted use, distribution, and reproduction in any medium, provided you give appropriate credit to the original author(s) and the source, provide a link to the Creative Commons license, and indicate if changes were made. The Creative Commons Public Domain Dedication waiver (http://creativecommons.org/publicdomain/zero/1.0/) applies to the data made available in this article, unless otherwise stated. 


\section{Background}

Brachyspira hyodysenteriae (B. hyodysenteriae) - a $\beta$ haemolytic Gram-negative oxygen-tolerant anaerobic spirochete - is the primary cause of swine dysentery, characterized by bloody to mucoid diarrhea due to mucohaemorhagic colitis is pigs [1]. Swine dysentery primarily affects pigs during the growth and finishing period. Transmission of $B$. hyodysenteriae occurs through the fecal-oral route and is associated with several risk factors such as introduction of colonized animals (carriers), poor external (quarantine, rodents, wild birds and other potential reservoirs) and internal biosecurity measures (adequate cleaning and disinfection protocols, mixing of age groups) [2]. Clinical signs usually start with loss of appetite and mild, yellow to greycoloured diarrhea, further progressing to watery diarrhea with blood, mucus and pseudomembranes [3]. This results in economic damage due to growth losses, mortality, increased variation in pig weight and decreased feed conversion at farm level.

Control and prevention of $B$. hyodysenteriae mainly consists of administration of antimicrobial drugs, besides management and adapted feeding strategies [4]. Currently, no commercial vaccines against $B$. hyodysenteriae are available [1], although some experimental vaccines, such as bacterins, subunit vaccines and live attenuated strains have been evaluated [5]. Recently, B. hyodysenteriae has been reported as a worldwide re-emerging disease with an increasing number of isolates having decreased susceptibility to several crucially important antibiotics in the control of swine dysentery [5-15].

Overall, control and prevention of B. hyodysenteriae seems to become more challenging, due to the limited treatment options [2], the lack of effective preventive feeding strategies and the increased awareness on reduction of antimicrobial use in animal production [16].

Consequently, research focused on non-antibiotic alternatives to reduce bacterial infections in general and $B$. hyodysenteriae infections in particular becomes more prominent. Adapted feeding strategies, including a high dietary concentration of inulin, have proven to reduce the incidence of swine dysentery due to B. hyodysenteriae in grower pigs [17]. A citrus extract commercialized as raw material and used as feed additive showed relevant in vitro bacteriostatic and bactericidal activity against $B$. hyodysenteriae at relatively low concentrations of 32 and $128 \mathrm{ppm}$, respectively [18]. Among others zinc has been evaluated as a potential intervention to control B. hyodysenteriae. In vitro addition of either $\mathrm{ZnSO}_{4}$ or $\mathrm{CuSO}_{4}$ to the growth medium of $B$. hyodysenteriae caused complete inhibition of hemolytic activity in 3 culture cycles. Further research revealed that the inhibition of hemolysin was specifically mediated by $\mathrm{Zn}^{2+}$ [19]. A comparative study with $\mathrm{ZnSO}_{4}, \mathrm{Zn}$-methionine and $\mathrm{ZnO}$ only demonstrated a prophylactic effect of high concentrations of in-feed $\mathrm{ZnO}$ (2000 ppm or higher) against $B$. hyodysenteriae in a mouse challenge model for swine dysentery [20]. However, in 2017, the Committee for Medicinal Products of Veterinary Use concluded that the benefits of $\mathrm{ZnO}$ for the prevention of diarrhea in pigs do not outweigh the environmental risks of the product [21]. The recent withdrawal of the marketing authorization of $\mathrm{ZnO}$ by the European Commission limits the availability of effective alternatives to antimicrobial drugs. Consequently, there is a continuing need for new, effective, non-antibiotic innovations to further improve animal health and welfare and to help reducing economic losses due to $B$. hyodysenteriae infections in pigs.

Chelation of zinc with an organic molecule to form a $\mathrm{Zn}-\mathrm{Na}_{2}$-EDTA - instead of covalent binding of zinc to inorganic oxygen - reduces its environmental impact [22]. In addition, previous in vitro (unpublished data) and in vivo studies [22] have demonstrated that the $\mathrm{Zn}$ $\mathrm{Na}_{2}$-EDTA chelated complex - in relatively low concentrations - is potentially able to reduce adverse effects due to $B$. hyodysenteriae infections in pigs. An in vivo feasibility study demonstrated a positive effect of the zinc chelate to fecal quality and consistency, general clinical signs and average daily weight gain (ADWG) in $B$. hyodysenteriae-infected animals. Moreover, at the last treatment day, $B$. hyodysenteriae was not detectable by qPCR in most of the treated animals [22]. Based on these promising in vitro and in vivo results, the goal of the present study was to assess the efficacy of the zinc chelate, formulated as the Veterinary Medicinal Product Intra Dysovinol $499 \mathrm{mg} / \mathrm{ml}$ (ID; Elanco, Greenfield, IN) in the treatment of clinical signs due to B. hyodysenteriae infection in pigs under more challenging field conditions. For this purpose, the effect on excretion of $B$. hyodysenteriae was evaluated during and after a 6-day treatment period in two wean-to-slaughter units in the Netherlands.

\section{Materials and methods}

\section{Inclusion of farms and animals}

Two farms with clinical disease due to B. hyodysenteriae in grow/finisher pigs within two weeks preceding the start of the study or preventing clinical signs due to $B$. hyodysenteriae by strategic application of antimicrobial drugs (but showing relapse upon cessation of therapy) were included in this study. The clinical signs of swine dysentery in Farm 1 were mainly characterized by chronic diarrhea without very little presence of additions (mucus, necrotic material), occurrence of runt pigs and increased mortality. In Farm 2, the clinical signs of swine dysentery were more pronounced with bloody diarrhea, addition of 
mucus and necrotic material combined with retarded growth and finally mortality.

Pigs were not allowed to receive any preventive or curative antimicrobial drug for $B$. hyodysenteriae in the 10 days preceding Study Day (SD) 0 (first day of administration of ID). Farms were representative for Dutch commercial farms housing grow/finisher pigs under the highest welfare conditions (three stars within the welfare concept) with the possibility to realize administration of ID via drinking water using a dosing pump. Animals were fed dry feed and no increased levels of zinc or copper via the feed or drinking water were allowed. Pens included in the study had identical stocking density per pen, feed, climate and management.

A pen was included when at least $10 \%$ of the animals in the pen were qPCR-positive for B. hyodysenteriae at SD -3 and at least one of the pigs was showing a nonnormal fecal score (score 1 or higher on at least one aspect as described below). Only post-weaned pigs, showing non-normal fecal scoring and excreting $B$. hyodysenteriae at SD - 3 and/or at SD0 or SD2 (the latest) were included in the study for individual follow-up within selected pens.

\section{Administration of ID}

Intra Dysovinol $499 \mathrm{mg} / \mathrm{ml}$ (Elanco) consists of $499 \mathrm{mg}$ of $\mathrm{Zn}-\mathrm{Na}_{2}$-EDTA per $\mathrm{ml}$ as an active ingredient in an aqueous solution further containing colorants and a preservative. Upon inclusion, the pen was randomly assigned to control or treatment with ID at a dosage of $0.023 \mathrm{ml}$ product per $\mathrm{kg}$ bodyweight for the duration of 6 days (according to SPC specifications), starting at SD0 and ending at SD6 (Table 1). Based on the total bodyweight and total water consumption of all animals in a single pen, a 100 times concentrated pre-dilution was dosed at $1 \%$ to the drinking water using a calibrated dosing pump.

\section{Clinical observations and fecal quality}

All animal observations and collection of animal samples were carried out by the observer. At pen level, general

Table 1 Study outline indicating the actions performed on the different study days. I, individual level; P, pen level

\begin{tabular}{|c|c|c|c|c|c|c|c|c|c|c|c|}
\hline \multirow[b]{2}{*}{ Parameter } & \multirow[b]{2}{*}{ Level } & \multicolumn{10}{|c|}{ Study day } \\
\hline & & -3 & 0 & 1 & 2 & 3 & 4 & 5 & 6 & 10 & 14 \\
\hline $\begin{array}{l}\text { General health } \\
\text { observations }\end{array}$ & $P$ & $x$ & $x$ & $x$ & $x$ & $x$ & $x$ & $x$ & $x$ & $x$ & $x$ \\
\hline Treatment & $P$ & & $x$ & $x$ & $x$ & $x$ & $x$ & $x$ & $x$ & & \\
\hline Clinical observations & । & & $x$ & $x$ & $x$ & $x$ & $x$ & $x$ & $x$ & $x$ & $x$ \\
\hline Faecal quality & । & & $x$ & & $x$ & & $x$ & & $x$ & & $x$ \\
\hline qPCR faeces & । & & $x$ & & $x$ & & $x$ & & $x$ & & $x$ \\
\hline Weighing & I & & X & & & & & & X & & X \\
\hline
\end{tabular}

health observations on all pigs in the selected pens were recorded from SD - 3 until to SD14 (Table 1). Pigs were individually identified by unique ear tag numbers. All individually identified pigs in a pen were weighed on SD0, SD6 and SD14. Individual clinical observations were conducted at SD 0, 1, 2, 3, 4, 5, 6, 10 and 14 and scoring for general clinical condition, alertness, lameness and signs of respiratory diseases. For evaluation of the fecal quality, scoring was performed at SD 0, 2, 4, 6 and 14 according to the scoring grid in Table 2. Fecal scoring included consistency, color and additions (mucus, foam, blood and necrotic material) and was added to obtain the total fecal score (TFS).

\section{Real-time PCR and quantitative real-time PCR}

Rectal fecal samples were collected at SD -3, 0, 2, 4, 6 and 14 in $50 \mathrm{ml}$ sterile vials (MLS nv, Menen, Belgium). At SD - 3, the samples were analysed for the presence of $\mathrm{B}$. hyodysenteriae by Real-Time PCR based on the nox gene. At all other sampling time points, the same real-time PCR was used combined with a standard curve in order to obtain a quantitative result (= quantitative real-time PCR). The cutoff $\mathrm{Ct}$ value is 37. For PCR analysis, DNA was extracted from $1 \mathrm{~g}$ of each individual faeces sample. Extraction was performed with the MagAttract 96 Cador Pathogen Kit (Indical Bioscience, Leipzig, Germany) according to the manufacturer's instructions. Per $\mathrm{g}$ feces, $5 \mathrm{ml}$ physiological solution was added to the sample, after which the mixture was thoroughly vortexed and $400 \mu \mathrm{l}$ of supernatant was collected for extraction. The real-time PCR was performed using the BactoReal Kit B. hyodysenteriae (Ingenetix, Vienna, Austria), which was based on the

Table 2 Fecal quality scoring grid for assessment of individual fecal quality. Sum (total fecal score, TFS) of fecal characteristics $A, B$ and $C$ was used for statistical analysis

\begin{tabular}{lll}
\hline Parameters & Score & Description \\
\hline A. Faecal shape and consistency & 1 & $\begin{array}{l}\text { Hard, dry, can be shaped } \\
\text { by fingers }\end{array}$ \\
& 2 & $\begin{array}{l}\text { Normal, soft, but not } \\
\text { watery } \\
\end{array}$ \\
& 3 & Pasty, soft \\
& 4 & Watery \\
B. Faecal color & 0 & $\begin{array}{l}\text { Normal dark green, brown, } \\
\text { feed color }\end{array}$ \\
& 1 & $\begin{array}{l}\text { Light brown to yellow } \\
\text { Grey }\end{array}$ \\
& 2 & Dark brown to black \\
C. Faecal additions & 3 & Normal, no additions \\
& 0 & Slimy or foamy \\
& 1 & Bloody or fibrinous exudate \\
\hline
\end{tabular}


nox gene of B. hyodysenteriae, using the ABI7500 detection system (Thermofisher, Massachusetts, USA) with the following cycling conditions: $2 \mathrm{~min}$ incubation at $50^{\circ} \mathrm{C}$ and $20 \mathrm{~s}$ incubation at $95^{\circ} \mathrm{C}$ comprise the denaturation step, followed by 45 cycles of $95^{\circ} \mathrm{C}$ for $15 \mathrm{~s}$ and $60^{\circ} \mathrm{C}$ for $1 \mathrm{~min}$. The fluorescence data were collected during the $60^{\circ} \mathrm{C}-1 \mathrm{~min}$ stage. The real-time PCR was based on TaqMan technology. A duplex PCR was performed using a probe labeled with the $\mathrm{FAM}^{\mathrm{mi}}$ dye for the target gene and a probe labeled with the $\mathrm{Cy}^{\circ} 186$ dye for the internal positive control (IPC). This IPC was used to check for PCR inhibition. PCR detection limit was 18 copies of nucleic acid (i.e. $1.26 \log _{10} \mathrm{cfu} / \mathrm{g}$ feces) per PCR reaction.

For quantification, the quantitative real-time PCR (qPCR) standard B. hyodysenteriae (Ingenetix, Vienna, Austria), containing $10^{7}$ copies $/ \mu$ l was used. Standard curves were performed using four dilution points: $10^{6}$ $10^{5}-10^{4}-10^{3}$. Efficiency was between 85 and $115 \%$ and $R^{2}$ was at least 0.99 . Based on these standard curves, $\mathrm{Ct}$ values were translated into $\log _{10} \mathrm{cfu} / \mathrm{gram}$ feces $(\mathrm{cfu}=$ colony forming unit).

\section{Concomitant therapeutic treatment and unexpected death}

If a pig enrolled in the study developed an abnormal health condition - such as profuse diarrhea, coughing or lameness - an individual therapeutic treatment was determined. In case an animal died or had to be euthanized because of severe illness/welfare reasons before SD0, the animal was disposed through regular channels without further investigation. In case of death/euthanasia after the treatment with ID or placebo had started, a fecal sample was collected for qPCR analysis (either immediately before euthanasia or as soon as possible after the pig was found dead) and examined post-mortem or disposed through regular channels.

\section{Justification of sample size}

Sample size calculations for a two-sided two-sample ttest were carried out in JMP 14.1 using the following parameters based on a previous field study with ID in the Netherlands [22]:

- Observed standard deviation of $2 \log _{10} \mathrm{cfu} / \mathrm{g}$ feces between both groups

- Calculations were performed using following statistical parameters:

- Alpha 0.05

- Power 95\%

- Minimal difference to be detected of $2 \log _{10} \mathrm{cfu} / \mathrm{g}$ feces of B. hyodysenteriae
Based on these calculations, 60 pigs per treatment group was sufficient and therefore, 120 animals were enrolled in the entire study, equally distributed over 2 farms.

\section{Statistical analysis}

The effect of treatment is tested for each time separately. To account for the variability between farms, the binary outcomes were analysed with a logistic regression model with treatment and farm as factors. The other outcomes were analysed with a linear regression model, again with treatment and farm as factors. All model parameters were estimated with the maximum likelihood method and the hypothesis tests were performed as Wald tests, which are for the linear regression model equivalent to the least squares method and $\mathrm{t}$-test, respectively. The $P$ values (one for each time) were adjusted with the Bonferroni method so as to control the familywise error rate (FWER). All tests were performed at the nominal FWER level of $5 \%$.

\section{Results}

Farms and animals

In total 122 pigs in 2 farms and 9 pens have been enrolled in the study for close, individual follow-up. Five of the initially selected pigs did not meet the inclusion criteria as they showed no excretion of $B$. hyodysenteriae at SD0. Therefore, data from 117 study pigs have been used to assess the efficacy of ID in the treatment of clinical signs due to $B$. hyodysenteriae infection under field conditions in the Netherlands. Of the 117 enrolled pigs, 60 received ID treatment ( 2 pens on farm A, 2 pens on farm $B$ ) and 57 received no treatment and were considered control (3 pens on farm A, 2 pens on farm B). On farm $\mathrm{A}$, the individually followed pigs had an average bodyweight of $37.7 \mathrm{~kg}$ (range: $24.1-54.9 \mathrm{~kg}$ ) on inclusion, while the pigs on farm B were younger with an average bodyweight of $15.2 \mathrm{~kg}$ (range: 9.4-22.4 kg). Bodyweights, distribution of sex (female/male), level of B. hyodysenteriae excretion and fecal quality score did not differ significantly at SD0 between ID-treated and control pigs as evaluated by the median test $(P>0.05)$ and the proportion test $(P>0.05)$, respectively.

\section{Clinical observations and fecal quality}

The general clinical score of the individually monitored pigs was comparable between ID-treated pigs and control pigs at SD0, with 25 animals in both group scoring normal (score 0). Following ID treatment, a rapid increase in the percentage of animals with a normal general clinical score occurred with $90 \%(54 / 60)$ of the animals scoring normal at SD6 (Fig. 1). In the control pigs, this general clinical score improved more slowly over time, resulting in $73.6 \%$ (42/57) of animals scoring 


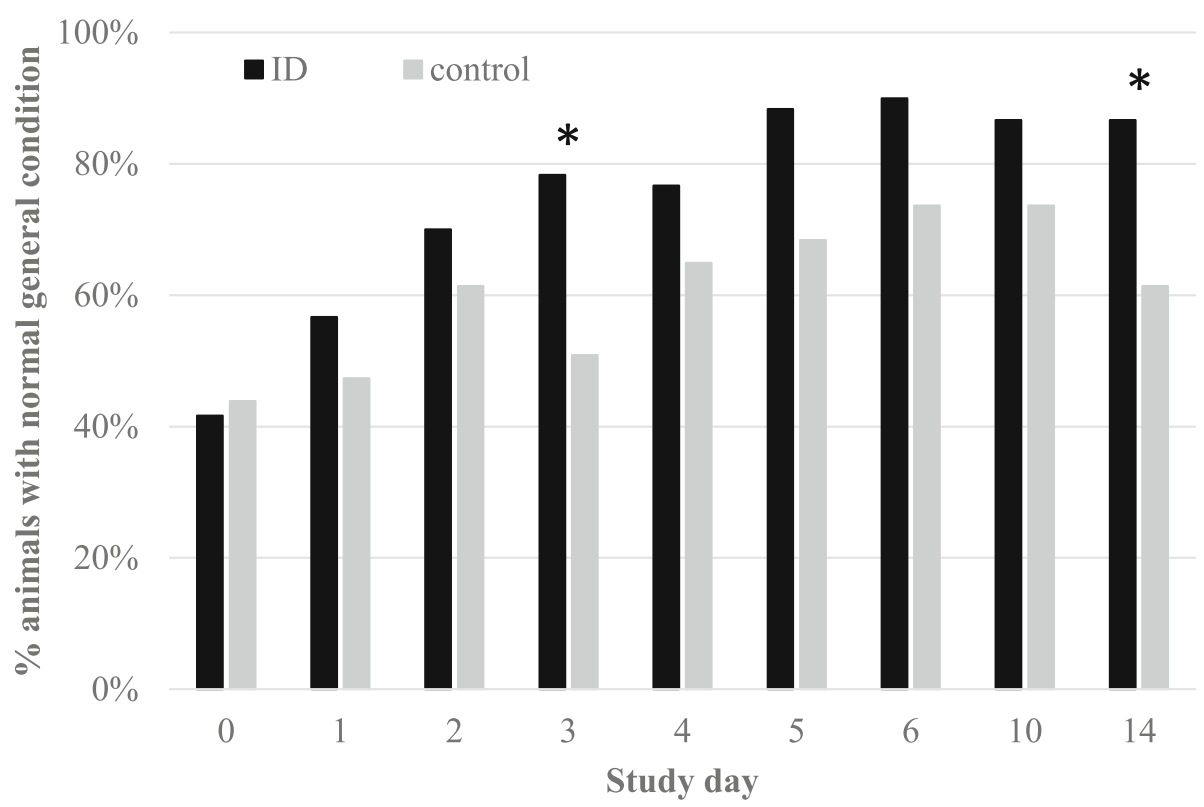

Fig. 1 Percentage of ID-treated $(n=60)$ and control $(n=57)$ pigs with a normal general clinical score per study day from SD 0 to 14 . Pigs were treated with ID from SD 0 to 6 . Significant differences $(P<0.05)$ are indicated with asterix*

normal at SD6. Following the end of ID treatment, the percentage of ID-treated pigs with a normal general clinical score remained relatively stable $(\sim 86.7 \%)$, while the percentage of control pigs with a normal general clinical score decreased significantly at SD14 $(P<0.05)$. The percentage of pigs with a normal general clinical score was significantly different $(P<0.05)$ between both groups from SD4 until SD14.
The total fecal score (TFS) of the individually monitored pigs was comparable between ID-treated pigs and control pigs on SD0 with an average score of 3.16 and ranging from 0 to 7 for both treatment groups (t-test, $P>0.05$ ). At SD0, TFS was numerically high in IDtreated pigs, however, from SD2 to SD6, ID-treated pigs had a lower TFS as compared to the control pigs $(P>$ 0.05; Fig. 2). At SD14, 8 days after the end of the ID

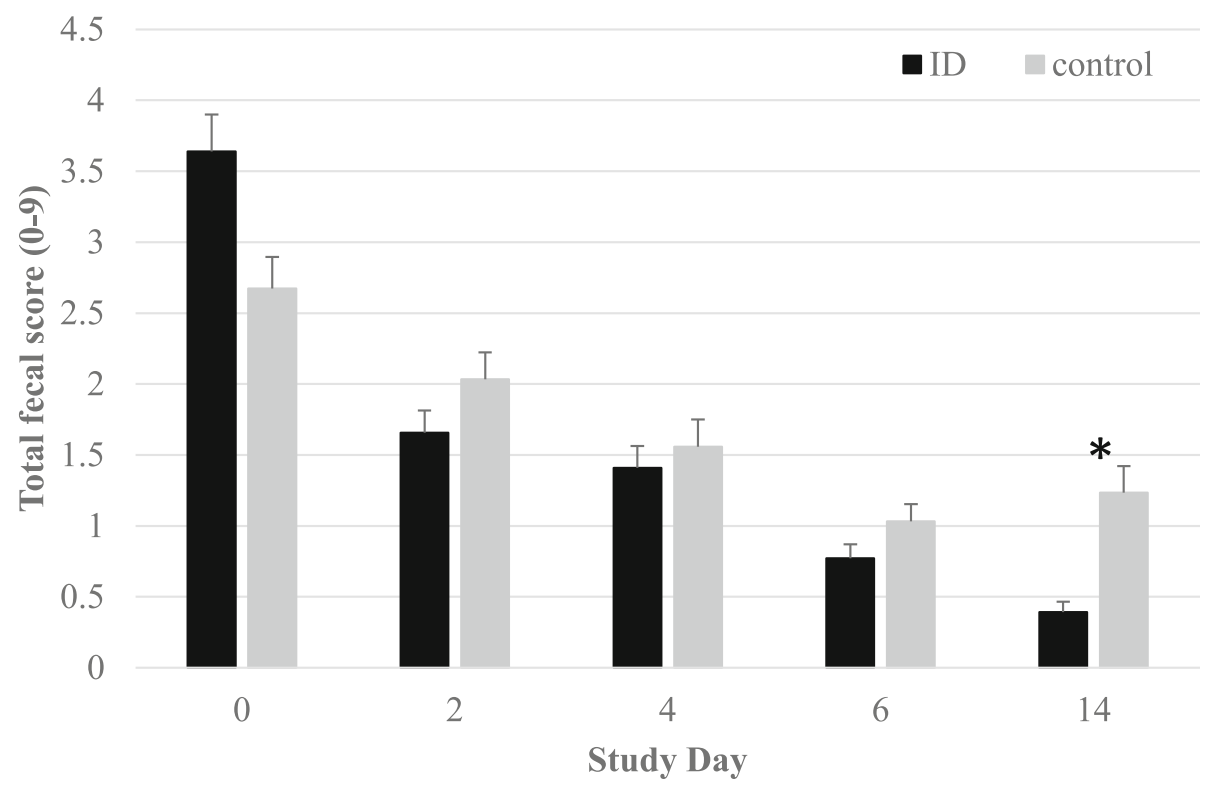

Fig. 2 Total fecal score (average \pm SEM) of ID-treated and control pigs per study day from SD 0 to 14. Pigs were treated with ID from SD 0 to 6. Significant differences $(P<0.05)$ are indicated with asterix* 
treatment, TFS in ID-treated animals (TFS 0.39) remained significantly lower $(P<0.01)$ as compared to control pigs (TFS 1.23), which was mainly due to differences in the scores of fecal consistency and fecal color. Overall, the ID-treated pigs showed an average daily improvement of their TFS by 0.74 , whereas TFS in control pigs only had an average daily improvement of 0.38 . Moreover, an increase in TFS was observed in control pigs at SD14. Overall, at SD6, 25 of the 60 ID-treated pigs showed a TFS of zero compared with 20 out of the 57 control pigs. At SD14, the number of ID-treated pigs with a TFS of zero increased to 39 out of 60 , while in control pigs, this number remained at 20 .

\section{Real-time semi-quantitative PCR}

Brachyspira hyodysenteriae nucleic acid was present at comparable levels in both treatment groups $\left(6.68 \log _{10}\right.$ vs. $6.28 \log _{10} \mathrm{cfu} / \mathrm{g}$ feces for ID-treated and control pigs, respectively) in all fecal samples collected at SD0, as determined by qPCR (t-test; $P>0.05$, Fig. 3 ). At SD0, 2 out of 60 ID-treated pigs were $B$. hyodysenteriae-negative, while none of 57 control pigs were PCR-negative for the pathogen (Fig. 4). The number of B. hyodysenteriae-negative pigs in the ID-treated group remained equal to the control group at SD2, but increased to a maximum of 40 out of 60 at SD4. A slight fall-back in the number of PCR-negative IDtreated pigs (35/60) occurred at SD6, although their bacterial load (expressed as $\log _{10} \mathrm{cfu} / \mathrm{g}$ feces) decreased to 2.21 at SD6. Univariable analysis showed that $B$. hyodysenteriae nucleic acid excretion was significantly reduced in IDtreated pigs from SD4 to SD14 as compared to control pigs (t-test, $P<0.05)$. Multivariable analysis showed that the overall $\log _{10} \mathrm{cfu} / \mathrm{g}$ feces decreased significantly in IDtreated pigs from SD0 to SD6 compared to control pigs: ID-treated pigs showed a $4.48 \log _{10} \mathrm{cfu} / \mathrm{g}$ feces reduction from SD0 to SD6, whereas control pigs only had a 0.63 $\log _{10} \mathrm{cfu} / \mathrm{g}$ feces reduction over the same period $(P<0.05)$.

\section{Average daily weight gain}

The ID-treated pigs that were individually monitored had a significantly higher ADWG (ADWG, g/day) throughout the entire study as compared to the control pigs (Fig. 5). During the treatment period (SD0-6), ADWG in ID-treated pigs was at $825 \mathrm{~g} /$ day $( \pm 61 \mathrm{~g} / \mathrm{d}$; SEM), while control pigs grew only $619 \mathrm{~g} / \mathrm{d}( \pm 63 \mathrm{~g} / \mathrm{d}$; SEM). In the period following the end of ID treatment (SD6-14), ID-treated pigs increased to an ADWG of $903 \mathrm{~g} / \mathrm{d}(( \pm 57 \mathrm{~g} / \mathrm{d} ;$ SEM$)$, whereas ADWG in control pigs decreased to $505 \mathrm{~g} /$ day $( \pm 59 \mathrm{~g} / \mathrm{d}$; SEM).

\section{Concomitant therapeutic treatment, unexpected death and adverse events}

Due to persisting severe clinical symptoms of B. hyodysenteriae infection in 20 control pigs (35\%), it was necessary to perform additional treatment with a single $(n=$ 17) injection of tiamulin (Denagard 10\%; Elanco) during the study. Three pigs needed more than one injection, namely two pigs were injected 3 times and one pig needed in total 4 injections of tiamulin. The ID-treated pigs did not require any additional treatment throughout the entire study and no mortality was observed in both treatment groups between SD0 and SD14. No adverse

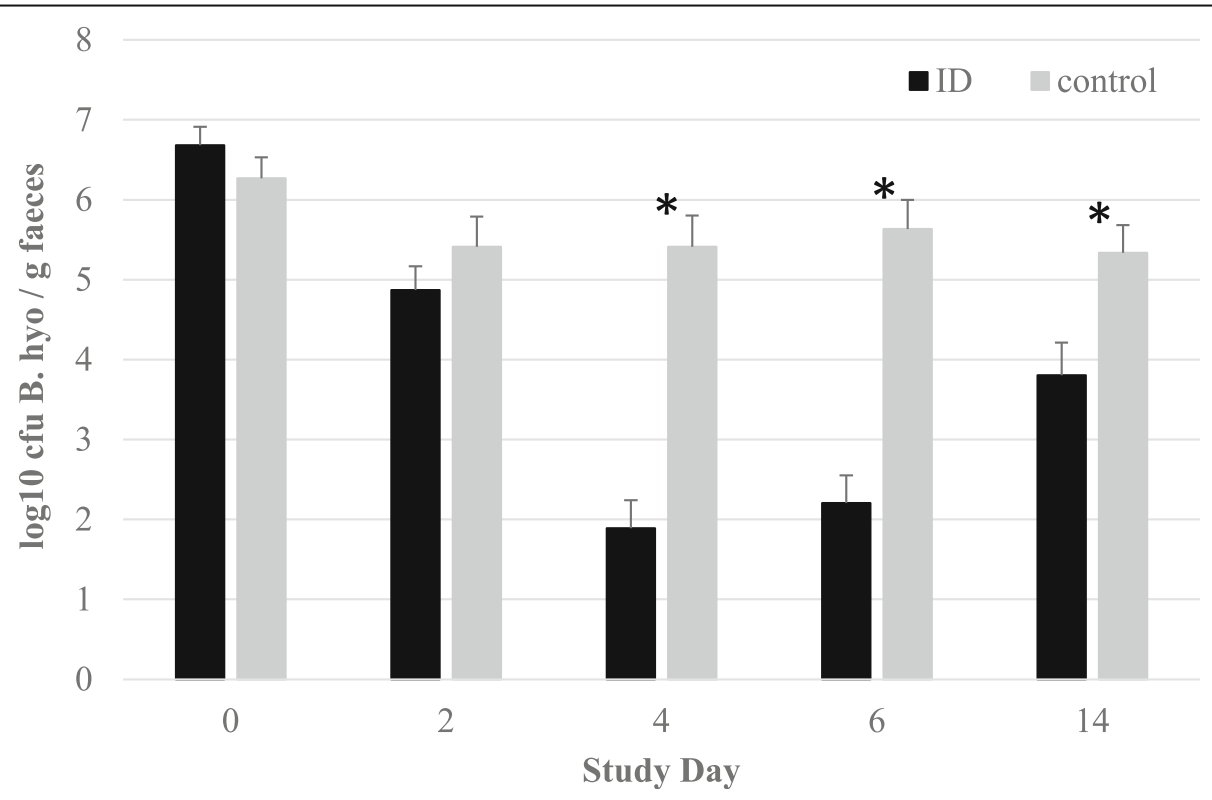

Fig. $3 \log _{10}$ cfu B. hyodysenteriae per $\mathrm{g}$ of feces (average \pm SEM) of ID-treated and control pigs per study day from SD 0 to 14. Pigs were treated with ID from SD 0 to 6 . Significant differences $(P<0.05)$ are indicated with asterix* 


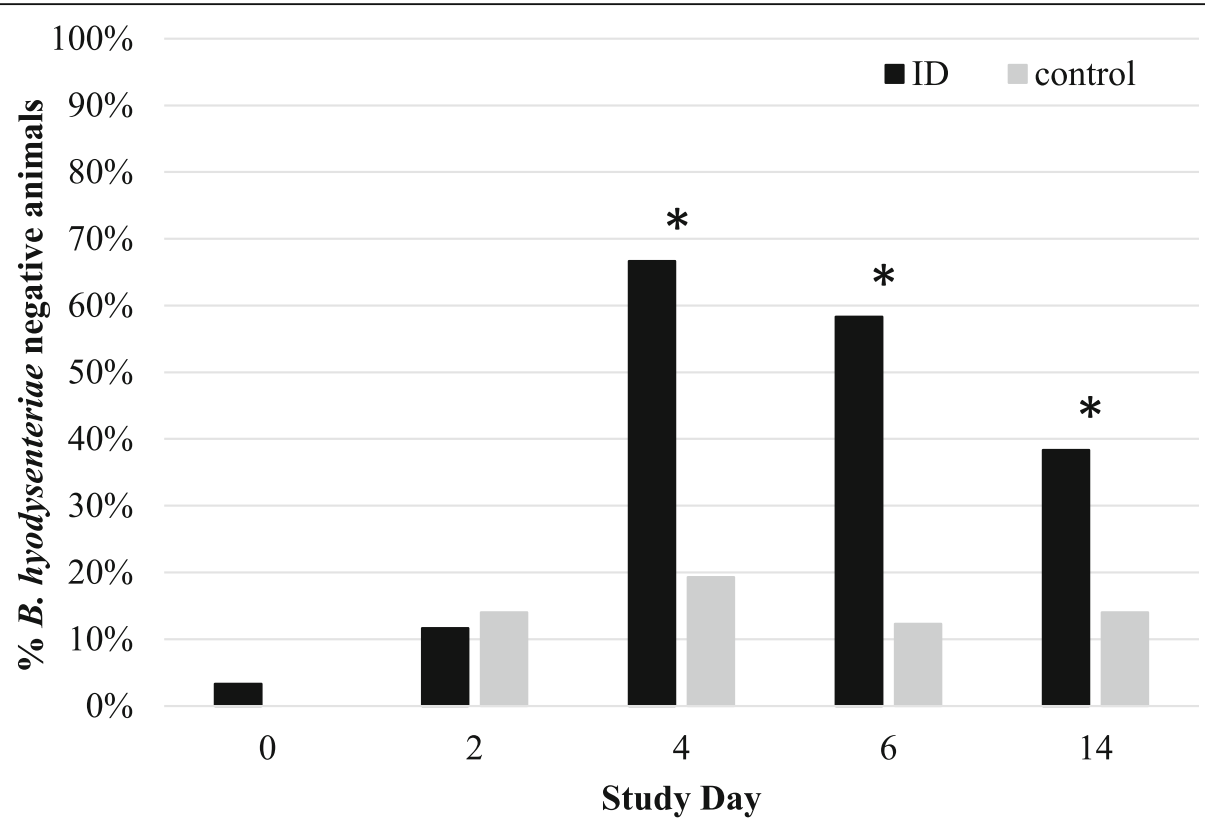

Fig. 4 Percentage of B. hyodysenteriae PCR-negative ID-treated and control pigs per study day from SD 0 to 14 . Pigs were treated with ID from SD 0 to 6 . Significant differences $(P<0.05)$ are indicated with asteri $x^{*}$

events related to ID treatment were observed during and after the study.

\section{Discussion}

The current study demonstrated that treatment with zinc chelate (Intra Dysovinol $499 \mathrm{mg} / \mathrm{ml}$; ID, Elanco) significantly reduced general clinical signs at SD3 and SD14, while shedding of B. hyodysenteriae was reduced with $4.48 \log _{10} \mathrm{cfu}$ per $\mathrm{g}$ feces during the treatment period, resulting in $58.3 \%$ B. hyodysenteriae negative animals on SD6. Treatment with ID for 6 consecutive days significantly improved TFS, which is an additive score of fecal consistency, color and eventual additions (mucus, foam, blood and necrotic material). The fecal quality remained good after the end of the 6-day ID treatment, although at SD14 an increase in B. hyodysenteriae-

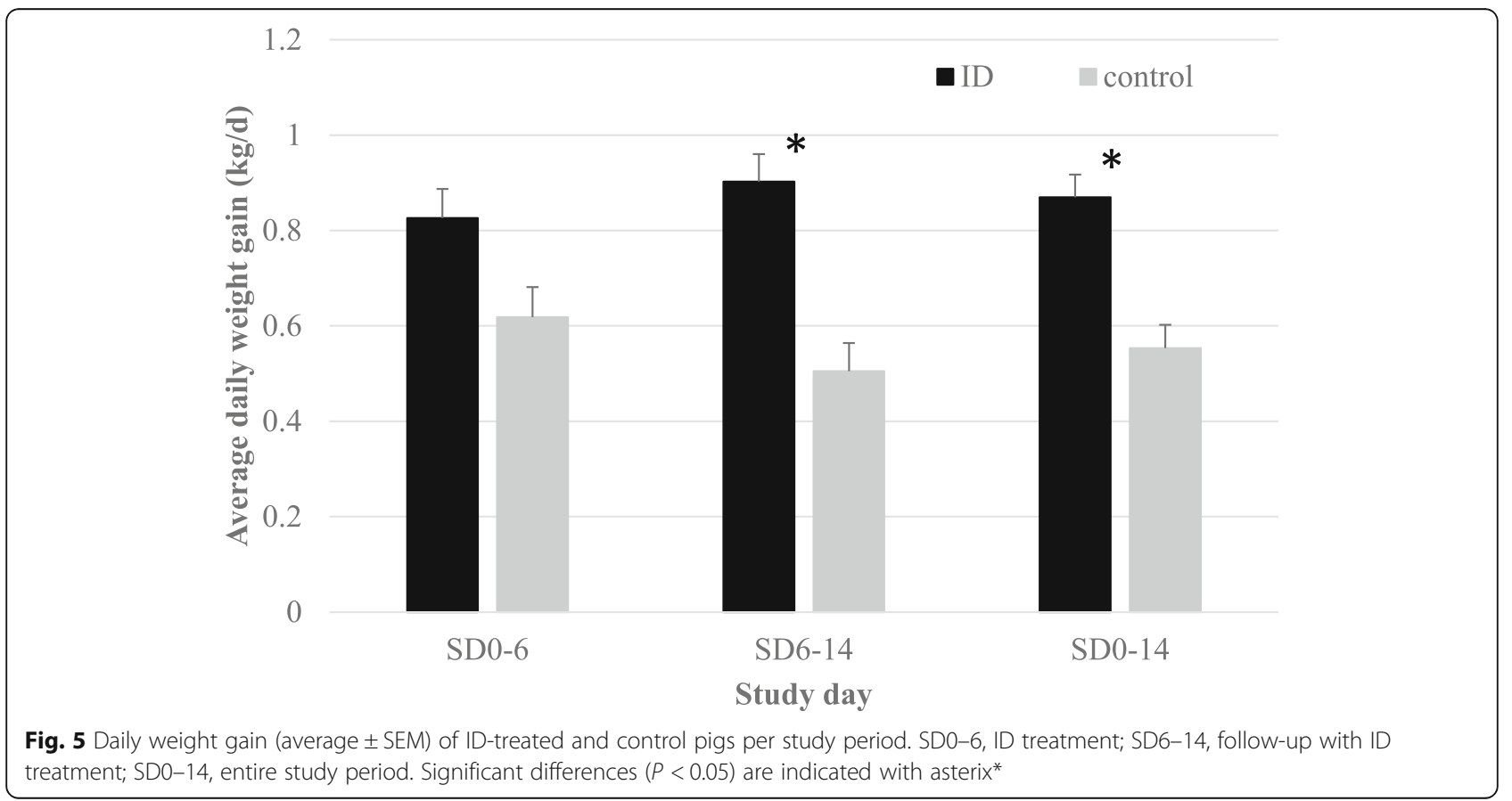


positive ID-treated pigs could be observed. This observation might be associated with the challenging housing circumstances of the pigs in the current study. In contrast to Lammers et al. (2019) [22], who performed his trials under conventional Dutch housing conditions, the pigs in our trial were housed in a concept including a high-welfare environment, which implicates more than $80 \%$ solid flooring, partly (50\%) bedded with straw, and only a very little slatted surface. These circumstances do not favour the evacuation of B. hyodysenteriae-infected feces throughout the study and might expose the pigs to moderate to high amounts of potentially contagious fecal material. Moreover, a high percentage of pigs consumed liquid fecal material present on the solid pen floors.

In the current study, $B$. hyodysenteriae excretion did not completely disappear at SD6 in contrast to the results reported by Lammers et al. (2019) [22]. Besides the above mentioned factors related to differences in housing and environmental infection pressure, basic differences in sampling approach and subsequent qPCR analysis were present. First, the fecal samples in the current study were collected as a rather large volume of feces in a sterile vial, which allows the analytic laboratory to weigh $1 \mathrm{~g}$ of feces from each vial for DNA extraction and subsequent qPCR analysis. In contract, E-swabs were used by Lammers et al. (2019) [22], which implicates that potentially less than $1 \mathrm{~g}$ of fecal material was available for extraction. This difference in sampling approach might already influence the diagnostic sensitivity of the qPCR. Secondly, the PCR cut-off Ct value of 40 corresponded with a limit of detection and limit of quantification of $2.90 \log _{10} \mathrm{cfu} / \mathrm{g}$ feces in the study by Lammers et al. (2019) [22], whereas in our study, the cut-off Ct value of 40 corresponded to $1.26 \log _{10} \mathrm{cfu} / \mathrm{g}$ feces. Taken together, these differences in sampling and analysis might at least partially explain the observed difference in percentage of $B$. hyodysenteriae-negative animals between both studies at SD6.

The severity of the B. hyodysenteriae infection in both farms required additional veterinary intervention in the untreated control pigs. Before the start of the study, antimicrobial sensitivity to tiamulin was checked for the B. hyodysenteriae strains isolated and tiamulin MIC was at $0.25 \mu \mathrm{g} / \mathrm{ml}$ in both farms. Overall, $35 \%$ of the control pigs was administered one or more additional therapeutic treatments, whereas none of the ID-treated pigs required additional therapeutic intervention.

Although bodyweight of the animals at SD0 was different between farm A and B, no farm effect is expected on the results, since both treatments were equally distributed between both farms, resulting in similar bodyweights for both treatment groups. The fecal quality improved from 2 days after treatment onwards and continued TFS improvement was observed until 8 days after the end of ID treatment. This rapid improvement in clinical signs after ID treatment was in line with the 100 -fold reduction in B. hyodysenteriae shedding within 2 days of ID treatment to an almost 10,000-fold reduction at 4 days in treatment. Although fecal scores continued to improved at SD6 and SD14, the qPCR results indicated a slight increase in B. hyodysenteriae shedding, which might be explained by the high environmental infection pressure due to the specific housing conditions. Considering the limit of detection by Lammers et al. (2019) [22], which was at $2.90 \log _{10} \mathrm{cfu} / \mathrm{g}$ feces, our current qPCR results could also be considered 'negative' for B. hyodysenteriae shedding at the cut-off level of $\mathrm{Ct}$ value 40 . Therefore, based on the clinical signs and fecal quality, we can conclude that the zinc chelate product had a sufficient efficacy in the treatment of swine dysentery due to $B$. hyodysenteriae.

The ability of $B$. hyodysenteriae to colonise the large intestine and its specific virulence factors are still not fully elucidated [2]. However, hemolysins, flagella, lipooligosaccharides and bacterial chemotaxis have been highlighted within the pathogenesis of swine dysentery, besides specific virulence life-style factors, such as outer membrane proteins, NADH oxidase and proteins of iron metabolism [1]. It requires further investigation which mechanism causes the novel zinc chelate to apparently prevent colonization and subsequently enhance the elimination of the pathogen [22] In a mouse model for swine dysentery, the effect of zinc methionine, $\mathrm{ZnO}$ and $\mathrm{ZnSO}_{4}$ has been assessed and only $\mathrm{ZnO}$ levels of at least $2000 \mathrm{ppm}$ demonstrated a prophylactic effect against $B$. hyodysenteriae, which is a considerably higher dose than what was required for the zinc chelate in the current study [20]. Another study reported no therapeutic effect of 250 ppm zinc chelate in the drinking water for 17 days to pigs inoculated with $B$. hyodysenteriae, which may be due to the nature of the chelating agent used [23].

Besides its impact on animal health and welfare, swine dysentery due to $B$. hyodysenteriae has a tremendous impact on the economic impact of an affected farm due to reduced pig performance, increased antimicrobial treatment costs and mortality. Annual losses of about $€ 133$ per sow were calculated for fattening pigs affected by clinical swine dysentery [24]. In the current study, growth results of the control pigs were significantly affected by swine dysentery, although we could not observe weight losses, in contrast to the study by Lammers et al. (2019) [22]. The ID treatment had a significant positive impact on pig performances with an overall ADWG of $869 \mathrm{~g} / \mathrm{d}$ from SD0 to SD14, while in the control pigs, the ADWG was only $553 \mathrm{~g} / \mathrm{d}$. These results indicate that the intestinal recovery at the level of the colon following ID treatment had a continued effect for at least 8 days after the end of 
ID treatment, which was clinically confirmed by the stable fecal quality and overall healthier appearance of the pigs in the ID-treated group.

Water medication is a convenient and flexible route of administration, permitting the farmer to apply the necessary treatment to a specific category of animals, resulting in an overall reduced use of therapeutics at farm level. Moreover, during a disease outbreak, water consumption remains stable for a much longer period as compared to feed intake, which implicates that disease animals can more efficiently be treated through this route of administration. During the trial, daily monitored water intake indicated that sick pigs continued to drink, while feed intake might be affected during the acute phase of $B$. hyodysenteriae infection [3].

\section{Conclusions}

Intra Dysovinol $499 \mathrm{mg} / \mathrm{ml}$ - containing a $\mathrm{Zn}-\mathrm{Na}_{2}-$ EDTA complex - is a non-antibiotic treatment for swine dysentery due to $B$. hyodysenteriae that reduces $B$. hyodysenteriae shedding with $4.48 \log _{10}$ cfu per g feces within its 6-day treatment. Treatment improved general clinical signs ( 90.0 vs. $73.6 \%$ animals with normal score in ID-treated vs. control) at SD6 and TFS (0.39 vs. 1.23 in ID-treated vs. control) at SD14 in naturally B. hyodysenteriae infected pigs. The positive effects of ID treatment remain for at least 8 days after cessation of oral ID therapy. Pigs remaining in a highly contaminated environment may be re-infected following the end of ID treatment, however, this is not different to standard antimicrobial therapy. Therefore, control of swine dysentery should combine an efficacious treatment with additional management practices to reduce the environmental infection pressure in order to limit re-infection as much as possible. The ID treatment resulted in a higher growth rate and improved general health, whereas no mortality was observed and no additional therapeutic treatments were necessary in contrast to the control pigs.

\section{Abbreviations}

ADWG: Average daily weight gain; B. hyodysenteriae: Brachyspira hyodysenteriae; cfu: Colony-forming unit; ID: Intra Dysovinol ; MIC: Minimal inhibitory concentration; qPCR: Quantitative polymerase chain reaction; SD: Study Day; SPC: Specified product characteristics; TFS: Total fecal score

\section{Acknowledgements}

The author greatly acknowledges both swine farmers and their swine veterinarians participating in the study.

\section{Author's contributions}

FV coordinated the entire study from study design to data collection and analysis to the manuscript. LA and EVD were responsible for laboratory coordination and analysis of fecal samples through $\mathrm{PPCR}$ for $B$. hyodysenteriae. RvB and GL were involved in protocol design and manuscript preparation. OT has been involved in statistical analysis. All authors read and approved the final manuscript.

\section{Authors' information}

FV is currently a Sr. Technical Advisor Swine for Benelux / UK\&ROI within Elanco Animal Health. He holds a DVM, a Master in Veterinary Public Health and Food Safety, a PhD in Veterinary Sciences and a PhD in Applied Biological Sciences, has a specific interest in swine intestinal health and the specific approach to improve intestinal health through non-antibiotic solutions.

\section{Funding}

The study was funded by Elanco Animal Health, which facilitated the conduct of the field trial.

\section{Availability of data and materials}

The datasets analysed during the current study are available from the corresponding author on reasonable request.

\section{Ethics approval and consent to participate}

Field trial with Veterinary Medicinal Product approved for use in swine. No additional ethical approval needed. Consent to participate was obtained following full information of farmers on the protocol to be carried out.

\section{Consent for publication}

Not applicable.

\section{Competing interests}

The authors declare that they have no competing interests.

\section{Author details}

${ }^{1}$ Elanco, BU Food Animals, Plantijn en Moretuslei 1 - 3rd floor, 2018 Antwerpen, Belgium. ${ }^{2}$ DGZ-Vlaanderen, Industrielaan 29, 8820 Torhout, Belgium. Intracare BV, Voltaweg 4, 5466 AZ Veghel, The Netherlands. ${ }^{4}$ I-BioStat, Data Science Institute, Hasselt University, Campus Diepenbeek, Agoralaan gebouw D, 3590 Diepenbeek, Belgium. ${ }^{5}$ Department of Data Analysis and Mathematical Modelling, Faculty of Bioscience Engineering, Ghent University, Coupure Links 653, 9000 Ghent, Belgium. ${ }^{6}$ National Institute of Applied Statistics Research Australia (NIASRA), University of Wollongong, Northfields Ave, Wollongong, NSW 2522, Australia.

Received: 18 October 2019 Accepted: 17 December 2019

Published online: 16 January 2020

\section{References}

1. Alvarez-Ordóñez A, Martinez-Lobo FJ, Arguello H, Carvajal A, Rubio P. Swine dysentery: aetiology, pathogenicity, determinants of transmission and the fight against the disease. Int J Environ Res Public Health. 2013;10:1927-47.

2. Hampson DJ, Lugsomya K, La T, Phillips ND, Trott DJ, Abraham S. Antimicrobial resistance in Brachyspira - an increasing problem for disease control. Vet Microbiol. 2019:229:59-71.

3. Hampson DJ. Brachyspiral colitis. In: Diseaseas of Swine. Zimmerman JJ, Karriker LA, Ramirez A, Schwarz KJ, Stevenson GW. Eds. 10 ${ }^{\text {th }}$ edition. Ames, IA; Wiley-Blackwell; 2012:680-696.

4. Lobová D, Čížek A. Bactericidal efficacy of two disinfectants against Brachyspira hyodysenteriae and one feed supplement against $B$ hyodysenteriae and B pilosicoli Vet Med - Czech 2004a;49:156-160.

5. Mahu M, Pasmans F, Vranckx K, De Pauw N, Vande Maele L, Vyt P, Vandersmissen T, Martel A, Haesebrouck F, Boyen F. Presence and mechanisms of acquired antimicrobial resistance in Belgian Brachyspira hyodysenteriae isolates belonging to different clonal complexes. Vet Microbiol. 2017b;207:125-32.

6. Duinhof TF, Dierickx CM, Koene MGJ, van Bergen MAP, Mevius DJ, Veldman KT, van Beers-Schreurs HMG, de Winne RTJA. Multiresistentie bij Brachyspira hyodysenteriae-isolaten op een varkensvermeerderingsbedrijf in Nederland. Tijdschr Diergeneeskd. 2008;133:604-8.

7. Hidalgo A, Carvajal A, Vester B, Pringle M, Naharro G, Rubio P. Trends towards lower antimicrobial susceptibility and characterization of acquired resistance among clinical isolates of Brachyspira hyodysenteriae in Spain. Antimicrob Ag Chemother. 2011;55:3330-7.

8. Joerling J, Barth SA, Schlez K, Willems H, Herbst W, Ewers C. Phylogenetic diversity, antimicrobial susceptibility and virulence gene profiles of Brachyspira hyodysenteriae isolates from pigs in Germany. PlosOne. 2018;13: e0190928. 
9. Karlsson M, Aspán A, Landén A, Franklin A. Further characterization of porcine Brachyspira hyodysenteriae isolates with decreased susceptibility to tiamulin. J Med Microbiol. 2004;53:281-5.

10. Kirchgässner C, Schmitt S, Borgström A, Wittenbrink MM. Antimicrobial susceptibility of Brachyspira hyodysenteriae in Switzerland. Schweiz Arch Tierheilk. 2016;158:405-10.

11. Lobová D, Smola J, Čížek A. Decreased susceptibility to tiamulin and valnemulin among Czech isolates of Brachyspira hyodysenteriae. J Med Microbiol. 2004b;53:287-91.

12. Massacci FR, De Luca S, Cucco L, Tentellini M, Perreten V, Pezzotti G, Magistrali CF. Multiresistant Brachyspira hyodysenteriae shedding by pigs during the fattening period. Vet Rec. 2018. https://doi.org/10.1136/vr.104886.

13. Mirajkar NS, Davies PR, Gebhart CJ. Antimicrobial susceptibility patterns of Brachyspira species isolated from swine herds in the United States. J Clin Microbiol. 2016;54:2109-19.

14. Rugna G, Bonilauri P, Carra E, Bergamini F, Luppi A, Gherpelli Y, Magistrali CF, Nigrelli A, Alborali GL, Martelli P, La T, Hampson DJ, Merialdi G. Sequence types and pleuromutilin susceptibility of Brachyspira hyodysenteriae isolates from Italian pigs with swine dysentery: 2003-2012. Vet J. 2015:203:115-9.

15. Vyt P, Hommez J. Antimicrobial susceptibility of Brachyspira hyodysenteriae isolates compared with the clinical effect of treatment. Flem Vet J. 2006;75: 279-85

16. Speksnijder DC, Jaarsma ADC, van der Gugten AC, Verheij TJM, Wagenaar JA. Determinants associated with veterinary antimicrobial prescribing in farm animals in the Netherlands: a qualitative study. Zoonosis Publ Health. 2015;62:39-51.

17. Hansen CF, Hernández A, Mansfield J, Hidalgo A, La T, Phillips ND, Hampson DJ, Pluske JR. A high dietary concentration of inulin is necessary to reduce the incidence of swine dysentery in pigs experimentally challenged with Brachyspira hyodysenteriae. Br J Nutr. 2011;106:1506-13.

18. de Nova PJG, Carvajal A, Prieto M, Rubio P. In vitro susceptibility of Brachyspira hyodysenteriae to a commercial citurs fruit extract. Res Vet Sci. 2017;115:318-24.

19. Dupont DP, Duhamel GE, Carlson MP, Mathiesen MR. Effect of divalent cations on hemolysin synthesis by Serpulina (Treponema) hyodysenteriae: inhibition induced by zinc and copper. Vet Microbiol. 1994:41:63-73.

20. Zhang P, Carlson MP, Schneider NR, Duhamel GE. Minimal prophylactic concentration of dietary zinc compounds in a mouse model of swine dysentery. Animal Health Res Rev. 2001;2:67-74.

21. European Medicinal Agency. Questions and answers on veterinary medicinal products containing zinc oxide to be administered orally to foodproducing species. Outcome of a referral procedure under Article 35 of Directive 2001/82/EC (EMEAN/A/118). 2017:EMA/394961/2017.

22. Lammers $G$, van Berkel $R$, Roijackers $D$, Brouwer-Middelesch $H$, van Hout J. Treatment of clinical Brachyspira hyodysenteriae with zinc chelate in pigs: a blinded, randomised controlled trial. Vet Rec. 2019. https://doi.org/10.1136/ vetrec-2019-105523.

23. Šperling D, Čížek A, Smola J. Effect of zinc chelate and valnemulin for the treatment of swine dysentery in an experimental challenge study. Res Vet Sci. 2014;96:30-2.

24. Sjölund $M$, Zoric $M$, Wallgren P. Financial impact of disease on pig production. Part III - gastrointestinal disorders. Proceedings of 6th European Symposium on Porcine Health Management. Sorrento, Italy. 7-9 May 2014.

\section{Publisher's Note}

Springer Nature remains neutral with regard to jurisdictional claims in published maps and institutional affiliations.

Ready to submit your research? Choose BMC and benefit from:

- fast, convenient online submission

- thorough peer review by experienced researchers in your field

- rapid publication on acceptance

- support for research data, including large and complex data types

- gold Open Access which fosters wider collaboration and increased citations

- maximum visibility for your research: over $100 \mathrm{M}$ website views per year

At BMC, research is always in progress.

Learn more biomedcentral.com/submissions 